Itinéraires Itinéraires

Littérature, textes, cultures

2012-2 | 2012

Intime et politique

\title{
Le Journal de Virginia Woolf ou l'invention d'une voix « à soi »
}

Sandra Cheilan

\section{OpenEdition}

Journals

Édition électronique

URL : http://journals.openedition.org/itineraires/1111

DOI : 10.4000/itineraires. 1111

ISSN : 2427-920X

Éditeur

Pléiade

Édition imprimée

Date de publication : 1 novembre 2012

Pagination : $59-70$

ISBN : 978-2-336-00027-5

ISSN : 2100-1340

Référence électronique

Sandra Cheilan, «Le Journal de Virginia Woolf ou l'invention d'une voix « à soi » », Itinéraires [En ligne], 2012-2 | 2012, mis en ligne le 01 novembre 2012, consulté le 19 avril 2019. URL : http://

journals.openedition.org/itineraires/1111; DOI : 10.4000/itineraires.1111

\section{(ब) $\Theta \Theta$}

Itinéraires est mis à disposition selon les termes de la licence Creative Commons Attribution - Pas d'Utilisation Commerciale - Pas de Modification 4.0 International. 


\title{
Le Journal de Virginia Woolf ou l'invention d'une voix « à soi »
}

\begin{abstract}
Virginia Woolf used her diary as a personal testimony and analysis of history, where privacy and politics intertwined. The intimist style of her diary had a polemical dimension: it consisted of fighting against official political speeches of a patriarchal society. The speech from "inside" was a subversion of the official and dominant kind: it was a place of another type even marginal of political discourse. We will study three characteristics of Woolf's diarist writing as the invention of a new political view: the subjectivation of political facts, the disqualification of prevailing views, and the creation of a voice of "one's own," an independent voice. The diary updates a "feminist writing" of politics, in contradiction with ideologies and values of Woolf's contemporary society.
\end{abstract}

Keywords : diary, feminist writing, marginal discourse, authority Mots clés : journal intime, écriture-femme, marginalité, subversion, autorité

Virginia Woolf a toujours été concernée par les rouages politiques de son époque. Elle analyse, dans ses pamphlets, le fonctionnement d'une société qu'elle juge patriarcale et phallocentrique, elle y observe et déchiffre l'attitude des acteurs politiques - hommes d'État, hommes d'Église, hommes de justice -, avec leur représentativité, leurs symboles et leurs moyens de communication. Par ailleurs, elle intègre à sa fiction la parole politique telle qu'elle est incarnée dans les discours officiels, les textes de loi, et véhiculée par les journaux, la radio, ou dans les genres littéraires que sont l'essai et le roman historique. Et si elle répugne d'abord à investir le champ politique dans son journal intime ${ }^{1}$, à partir de 1936,

1. Elle refuse même de s'attarder sur la signature de paix de la Première Guerre mondiale : « One ought to say something about Peace day, I suppose, though wether its work taking a new nib for that purpose I don't know », Virginia Woolf, Diary, vol. I, New York, Harcourt, coll. «A Harvest Book », 1977, p. 292 (traduction de Colette-Marie Huet et de Marie-Ange 
son écriture révèle progressivement la nécessité et le devoir de témoigner de son époque, d'autant qu'elle vit personnellement les effets des événements. Son mari, Leonard Woolf, a été un membre actif du Labour Party ${ }^{2}$, ce qui a permis à la romancière d'être un témoin privilégié des réunions de parti et du fonctionnement des institutions politiques. Elle-même fut une militante du droit des femmes : elle donna de multiples conférences aux ouvrières et participa aux réunions de la Women's Guilde. Le double constat de l'absence du droit des femmes et de la montée des fascismes, associé à la mort tragique de son neveu Julian lors de la guerre civile espagnole, la pousse à intégrer la sphère publique au sein de l'espace intime qu'est son journal.

Woolf se saisit alors du journal personnel pour écrire sur et contre la politique. La poétique diariste croise espace du dehors et espace du dedans, moi social et moi profond, opérant un nivellement de la parole privée et du discours public, de l'intime et de la politique, tel qu'ils sont définis en conclusion de Trois Guinées. Tandis qu'elle enquête sur le rapport entre la peur « intime » et la « peur publique », l'image du dictateur inspire à Woolf une réflexion sur « un lien pour nous de la plus grande importance » :

Elle suggère que l'univers de la vie privée et celui de la vie publique sont inséparablement liés. Que les tyrannies et les servilités de l'un sont aussi les tyrannies et les servilités de l'autre [...]. Car telle sera notre ruine, si, dans l'immense espace abstrait de votre vie publique, vous oubliez l'image intime; ou si nous oublions dans l'immensité de nos émotions intimes, le monde extérieur et public. Nos deux maisons seront détruites, l'édifice public et la demeure privée, la matérielle et la spirituelle, car elles sont inséparablement liées ${ }^{3}$.

C'est dans le triple mouvement de subjectivation du fait politique, de disqualification des discours officiels et d'invention d'une voix « à soi », qui aspire au renouvellement de la société et des institutions, qu'il s'agira d'étudier l'écriture intimiste comme lieu de fabrique de la pensée politique woolfienne et d'une écriture qui lui est propre.

\section{Intimisation du politique et politisation de l'intime}

Dans le Journal, Woolf décloisonne la traditionnelle dichotomie entre vie publique et vie privée : histoire personnelle, histoire politique et mondiale et évolution de l'écriture s'entrecroisent. Ainsi le compte rendu

Dutartre) : «Sans doute me faudrait-il parler de la journée de la Paix, mais je ne suis pas bien sûre que l'occasion vaille la peine d'étrenner une plume neuve », Virginia Woolf, Journal intégral : 1915-1941, Édition intégrale, Paris, Stock, 2008, p. 272.

2. Leonard Woolf a été secrétaire au comité consultatif du Labour Party sur les questions internationales.

3. Virginia Woolf, Trois Guinées, trad. de l'anglais et préfacé par Viviane Forrester, Paris, 10/18, 2002, p. 227-228. 
des événements est-il filtré par l'intériorité de l'écrivain. La description du procès de Radclyffe Hall ${ }^{4}$ fait l'objet d'une satire kafkaïenne de l'absurdité juridique, la mort du roi est détournée au profit d'une description, lyrique et poétique, d'une Londres renaissante, et les actions de la politique internationale avec la « terrible ascension » de Hitler remettent en cause le moi anglican de Virginia qui perçoit, pour la première fois, son identité juive, par solidarité avec son mari, mais aussi avec toutes les victimes du totalitarisme. Si le politique investit progressivement l'intimité d'un sujet, dont l'essence émerge de sa confrontation avec l'État, actes et organisation politiques se trouvent déplacés et redéfinis dans le journal intime sur le mode de la résistance individuelle, de la liberté d'expression, et de la promotion de l'affect et de l'émotion comme valeurs nouvelles.

Cette interaction entre le politique et le moi aboutit à un traitement spécifique de la temporalité. Le temps long des mouvements de la société, celui de l'urgence de l'action et des décisions politiques contaminent le tissu répétitif et rituel du journal. L'écriture diariste est une écriture réactive, dans laquelle le temps de l'intime subit les aléas et les chocs des événements extérieurs, et où le moi s'en trouve modifié. À partir de mai 1938, Woolf recopie systématiquement les titres des journaux, l'événement politique servant alors de datation et d'impulsion au discours introspectif. Temps du politique, rythmé par les titres de la presse, et temps calendaire du journal viennent alors structurer une temporalité subjective aléatoire, assurant la continuité du texte et du moi. Participant d'une écriture de la notation, la prose journalistique, qui accumule les nouvelles, dramatise le temps subjectif sous forme de chocs, de surprises et de retournements : urgence politique et vibration intérieure se nouent dans un même " stream of consciousness ${ }^{5}$ », la date seule assurant la cohésion de cette écriture déliée. Le 17 septembre 1938, l'emploi du seul présent permet de creuser et de croiser les différents plans de réalité : on passe sans transition des nouvelles que donnent les journaux de l'avancée de la guerre et de la prochaine venue de Hitler en Angleterre à la description plus personnelle du rêve de Julian qui ne serait pas parti à la guerre, et de Roger qui ne serait pas mort. Ainsi, le présent de l'écriture fait coexister et oppose temps de l'actualité politique, temps du souvenir, temps du rêve qui est un contre-temps, celui du possible et de la

4. Radclyffe Hall est une poétesse et romancière britannique. Elle publia le roman lesbien Le Puits de solitude (The Well of Loneliness) en 1928, qui provoqua le scandale, et pour lequel elle fut attaquée en justice. Après un procès où elle fut accusée d'atteintes aux mœurs et d'obscénité, le livre fut censuré et interdit en Angleterre malgré la campagne de soutien de Virginia Woolf et de la maîtresse de cette dernière, Vita Sackville-West.

5. Cette expression peut être traduite en français par « courant de conscience », et désigne une technique littéraire, typique du roman moderniste, qui cherche à transcrire le flux et la complexité de la vie intérieure. L'expression a été inventée par William James dans The Principles of Psychology (1890) où il parle de « stream of thought, of consciousness, or of subjective life » pour désigner l'activité de l'esprit. 
réversibilité. C'est dans l'écart douloureux entre le présent inhumain et le hors-temps du rêve que se dit l'horreur de la guerre.

Dans «ce bizarre méli-mélo de choses publiques et de choses privées ${ }^{6} »$, l'identité individuelle est constitutive d'une conscience politique, construite sur la mémoire collective et des valeurs nationales. Dès lors, la mise en scène de différents moi contradictoires juxtaposés dans un temps fragmenté emblématise une conscience politique en perte de repères. Le moi de Woolf se donne à déchiffrer comme le microcosme de la crise des valeurs politiques et des certitudes tandis que l'histoire politique devient une trame essentielle et structurelle du journal. Témoignage de l'histoire présente, le journal est aussi le garant du souvenir, un " garde-mémoire ${ }^{7}$ » qui met en miroir histoire collective et histoire personnelle à travers les différentes formes de remémoration. Le 3 septembre 1928, l'auteur décrit une scène matricielle avec son père dans laquelle la mémoire personnelle remplace l'histoire nationale, l'expérience du père se substituant à l'apprentissage scolaire :

The Battle of Dunbar, the Battle of Worcester, \& the death of Cromwell - how often it seems to me I said that to my father ("my" father, not "father" any more) at St Ives; standing bolt upright in the dining room at Talland House ${ }^{8}$.

Il n'est pas anodin que le souvenir du père soit justement associé au souvenir des leçons d'histoire, mais d'une histoire masculine et officielle, celle de la guerre. Or ce souvenir personnel du passé de l'Angleterre est relayé par une scène domestique actuelle qui révèle une autre figure tutélaire, celle de la mère de Leonard. Ce glissement d'une autorité à l'autre aboutit à une réflexion plus générale sur le fonctionnement d'une société anglaise figée et castratrice, sur « cette tyrannie qu'exerce la mère - ou le père - sur la fille ${ }^{9} »$. Le texte donne ainsi à lire le mouvement d'intimisation de la politique : l'histoire de l'Angleterre est reconstituée à travers l'expérience personnelle, et le souvenir individuel prend la place de la mémoire collective. Ce que permet le journal, c'est bien une vision expérimentale et non dogmatique du fonctionnement sociétal. En effet, cette scène primitive,

6. Virginia Woolf, Journal intégral : 1915-1941, op. cit., p. 1286, traduction de « this odd mix up of public \& private», dans Virginia Woolf, Diary, vol. V, New York, Harcourt, coll. «A Harvest Book », 1984, p. 110.

7. Françoise Simonet-Tenant, Le Journal intime, genre littéraire et écriture ordinaire, Paris, Téraèdre, 2004, p. 116.

8. Virginia Woolf, Diary, vol. III, New York, Harcourt, coll. " A Harvest Book », 1980, p. 194, traduit par «La bataille de Dunbar, la bataille de Worchester et la mort de Cromwell... il me semble que j'ai récité cela d'innombrables fois à mon père ("Mon père", et non plus "Père" tout court) à St Ives, debout, raide comme un piquet, dans la salle à manger de Talland House », Virginia Woolf, Journal intégral, op. cit., p. 728.

9. Ibid., p. 729. 
qui associe l'histoire à la figure paternelle et au bellicisme, se charge d'une véritable résonance politique : elle exhibe l'histoire d'un moi écrasé par le poids de l'héritage paternel, symbole de l'autorité masculine qui gouverne l'Angleterre et qui mène aux pires barbaries et au nazisme, selon l'analyse que propose Virginia dans Trois Guinées.

Dans ce processus de subjectivation du politique, Woolf s'empare du motif de la grève, forme de la résistance pacifique qu'elle prône contre tout type d'action. Elle consacre un véritable témoignage aux journées de grève, figure du blocage, par opposition aux agitations vaines des hommes d'État. Ainsi écrit-elle le 5 mai 1926 :

An exact diary about the Strike would be interesting. For instance, it is now at $1 / 4$ to 2 : there is a brown fog; nobody is building; it is drizzling. The first thing in the morning we stand at the window \& watch the traffic in Southampton Row. This is incessant. Everyone is bicycling; motor cars are huddled up with extra people. There are no buses. No placards. no newspapers. The men are at work in the road; water, gas \& electricity are allowed; but at 11 the light was turned off ${ }^{10}$.

Le compte rendu objectif et scientifique des événements s'oppose à la saisie émotionnelle du vécu. Les phrases averbales, le recours aux présentatifs et la précision horaire relèvent du style lapidaire et haché de l'esthétique journalistique. Au contraire, les effets de répétition sonore de la syllabe finale « -ing » (« interesting », « building », « drizzling », « thing », «morning »), le recours au rythme ternaire (« no buses. No placards. no newspapers », « water, gas \& electricity »), et surtout la généralisation de la négation tant sur le plan syntaxique que lexical (le terme nobody signifie dans sa chair même les effets de la grève qui est négation du mouvement) relèvent d'une modulation affective de la phrase. Ici, l'analyse politique et sociale attendue de ce mouvement de grève est délaissée à tel point que la description sèche et inhabituelle des premières lignes pourrait être analysée comme une parodie de l'écriture froide et scientifique de l'histoire. Ces pages sont construites sur un système de tension et de paradoxes : l'écriture de notation vise à mimer les effets de la grève, notamment l'arrêt, là où sont narrativisés et mis en mouvement les sentiments intérieurs. D'ailleurs, le texte s'achève sur un fantasme inconscient, celui de Vita Sackville, avec laquelle Virginia entretient une relation à cette époque : «It is now

10. Virginia Woolf, Diary, vol. III, op.cit., p. 77, traduit par « Il serait intéressant de tenir un journal précis de la guerre. Par exemple, il est maintenant deux heures moins le quart. Il fait un brouillard brunâtre; aucun maçon ne travaille; il bruine. La première chose que nous faisons le matin, c'est de nous mettre à la fenêtre pour observer la circulation dans Southampton Road. Elle est continuelle. Tout le monde est à bicyclette. Des passagers supplémentaires s'entassent dans les automobiles. Il n'y a pas d'omnibus. Pas de placards; pas de journaux. Les ouvriers sont au travail sur la chaussée. L'eau, le gaz, l'électricité sont distribués; mais à onze heures la lumière a été coupée », Virginia Woolf, Journal intégral, op. cit., p. 628. 
chilly lightish evening; very quiet; the only sound a distant barrel organ playing ${ }^{11} »$. Dès lors, le blocage de la grève est dépassé par la mise en marche de la rêverie érotique et auditive de l'orgue de Barbarie, associée à Vita, et par la sensualité de l'écriture qui oppose à la négation de la grève la circulation des images et des sensations. Le déclenchement de l'écriture permet de dépasser une situation sociale et politique figée. Woolf met d'ailleurs en jeu et en mots les images obsessionnelles de sa cosmogonie personnelle et poétique : la voix, notamment masculine et autoritaire de la radio, la circulation, le temps suspendu, la promenade dans un Londres apocalyptique et désertique. Cette description se donne ainsi à lire comme une véritable mise en abyme : la position de Woolf spectatrice, placée derrière une fenêtre, relève de la posture de la femme écrivain, qui observe et recompose le spectacle de l'histoire à travers le filtre de son intériorité, matérialisée par la vitre. Woolf métamorphose, transfigure et intériorise la pratique et l'action politiques. La cristallisation des événements historiques autour d'images récurrentes et cette figure de la femme-écrivain mènent à une lecture subjectivée et mythifiée de l'actualité politique de son époque.

\section{De la subversion politique à l'invention d'une voix à soi}

La traditionnelle position de repli associée à l'intime revêt un caractère essentiellement polémique dans le journal woolfien : il s'agit de lutter contre les discours politiques officiels et la conception de la société qu'ils véhiculent. Woolf fait de l'écriture du dedans une forme de combat et de résistance intérieure au système institutionnalisé. Le journal circonscrit alors un «espace clandestin de liberté d'expression ${ }^{12}$ »: indépendance intérieure, liberté politique et invention formelle vont de pair et participent au renouvellement de la société auquel aspire la romancière. Comme le souligne Hélène Camarade, à propos des journaux intimes sous le Troisième Reich, le journal « se présente tout d'abord comme un domaine personnel où peut se développer l'autonomie de la pensée et de l'écriture : les diaristes y font valoir leur droit à l'intimité, à la liberté de conscience et à la liberté d'expression. Il s'agit d'un espace clandestin de libertés, d'un refuge intime qui permet de se soustraire, ne serait-ce qu'un instant, au système totalitaire $^{13} »$. Ainsi, en s'emparant des codes du journal, Virginia Woolf met en place un espace de subversion. Véritable contre-langage, l'écriture du journal saborde les genres canoniques et les symboles associés au politique dans une stratégie de dérision à l'encontre de l'autorité établie.

11. Virginia Woolf, Diary, vol. III, op.cit., p. 79, traduit par « Il fait maintenant un crépuscule glacial, lumineux, très calme; seul un orgue de Barbarie se fait entendre au loin », Virginia Woolf, Journal intégral, op.cit., p. 631.

12. Hélène Camarade, Écritures de la résistance : le journal intime sous le Troisième Reich, Toulouse, Presses Universitaires du Mirail, 2007, p. 62.

13. Ibid., p. 382. 
Ainsi en est-il du traitement des discours des hommes politiques, qu'il s'agisse de Chamberlain, de Hitler ou du prince Edward. Lors de la crise de la royauté, la diariste retranscrit le discours de ce dernier, prononcé le 10 décembre 1936 :

Then we had the Broadcast. "Prince Edward speaking from Windsor Castle" - as the emotional butler announced. Upon which, with a slight stammer at first, in a steely strained voice, as if he were standing with his back against the wall, the King (but that is already vanishing \& attaching itself to York) began: "At long last ... I can speak to you... The woman I love... I who have none of those blessings..." Well, one came in touch with human flesh, I suppose. Also with a set pigheaded steely mind... a very ordinary young man; but the thing had never been done on that scale. One man set up in the Augusta Tower at Windsor addressing the world on behalf of himself \& Mrs Simpson. [...] And then Edward went on in his steely way to say the perfectly correct things, about the Constitution, the P. Minister, her majesty my mother. Finally he wound up God Save the King with a shout ${ }^{14}$.

La crise des valeurs est traduite ici par une dégradation de l'autorité politique incarnée par la figure tutélaire du roi, satire qui se fixe sur la disqualification de son discours. Le jeu de citations tronquées par les points de suspension et la faiblesse de l'élocution du prince visent à montrer l'inanité de cette déclaration. Par ailleurs, la démystification du roi devenu personnage de quelque mélodrame, le décalage burlesque entre l'hymne national et les préoccupations matrimoniales révèlent l'effondrement de ce que Zweig appelait le «monde d'hier ». Loin du solipsisme attendu, le Journal apparait alors comme une œuvre dialogique et ironique : Woolf s'empare du discours autre, l'exhibe et le détourne, soit qu'elle le vide de son substrat idéologique en le tronquant, soit qu'elle joue de l'écart héroïcomique entre la prétention du discours et la situation. Or ce dialogisme vise aussi à détruire toute idéologie et à défier toute stigmatisation. Ainsi passe-t-on des discours officiels entendus à la radio aux gros titres froids des journaux, du point de vue de Freud sur la ruine de la civilisation aux opinions du petit peuple. Woolf fait entendre la cacophonie de voix et

14. Virginia Woolf, Diary, vol. V, op. cit., p. 43-44, traduit par «"Le prince Édouard vous parle depuis le château de Windsor", comme nous l'a annoncé le maître des lieux avec émotion. Sur quoi, avec un léger bégaiement au début, d'une voix tendue, inflexible, comme s'il parlait le dos au mur, le roi (mais cela n'est déjà plus de mise, le titre s'est attaché de lui-même à York) commença : “... Enfin... Enfin... je puis vous parler... La femme que j'aime... Je ne possède aucun de ces dons..." Enfin on atteint là au vif de la nature humaine, je suppose et on a affaire aussi à un esprit d'acier, absolument déterminé, à ... un jeune homme très ordinaire; mais la chose n'avait encore jamais pris cette dimension. Un homme seul, du haut de la Tour Augusta du château de Windsor, s'adressait au monde entier au sujet de Mrs Simpson et de lui-même. [...] Puis Edward poursuivit, disant exactement ce qui s'imposait au sujet de la Constitution, du Premier Ministre et de "sa majesté ma mère". Pour finir il dit dans un cri "God Save the King!" ", Virginia Woolf, Journal intégral, op.cit, p. 1227-1228. 
de points de vue discordants sans jamais conclure : devant ce verbiage absurde, le lecteur est amené à suspendre son jugement et à refuser toute fixation du sens.

La satire des hommes politiques passe aussi par des portraits caricaturaux où la métaphore théâtrale est récurrente. La guerre devient une véritable scène de théâtre sur laquelle les anciens héros de l'épopée ou du drame laissent place à des personnages fantoches, des postures fausses. Les assemblées du Labour Party se transforment en une mise en scène de soi : Lansbury prend la «pose » et « joue inconsciemment le rôle du chrétien persécuté ${ }^{15} »$. Par ailleurs, le commentaire des discours de Hitler préfigure la didascalie beckettienne avec sa gestuelle automatique et réifiée de quelque marionnette, absurde et bestiale : Woolf parle de « hurlement sauvage ", d' « aboiement », et décrit une véritable mise en scène : « Hitler a plastronné et tempêté », «il vient se planter tout au bord pour brailler des insultes ${ }^{16}$ ». Non seulement la réactivation du theatrum mundi contribue au discrédit des hommes politiques mais le brouillage entre fiction et réalité participe de la déréalisation de la guerre. La dérive fictionnelle sert alors à exprimer une incompréhension et une folie généralisées : le domaine politique devient une fiction théâtrale, il est littéralement incroyable et insensé.

$\mathrm{Au}$ cœur de cet inconcevable, ce qui se donne à lire, ce sont des bribes, des reliquats des différents grands genres qui servaient à valoriser le politique. Les séquences patriotiques et sublimes des romans historicoréalistes qui glorifiaient le peuple sont remplacées par les scènes intimistes, comme celle de la British Library racontée le 28 septembre 1938 : il s'agit d'un dialogue entre Virginia Woolf et « un bonhomme qui enlevait doucement la poussière ${ }^{17}$ » et qui s'inquiète de voir Virginia sans son masque à

15. Ibid., p. 1173, extrait traduit de «It was very dramatic: Bevin's attack on Lansbury. Tears came to my eyes as L[ansbury] spoke. And yet he was posing I felt - acting, unconsciously, the battered Christian man. Then Bevin too acted I suppose. He sank his head in his vast shoulders till he looked like a tortoise », Virginia Woolf, Diary, vol. IV, New York, Harcourt, coll. «A Harvest Book », 1982, p. 346.

16. Virginia Woolf, Journal intégral, op.cit., p. 1341, traduction de «Hitler boasted \& boomed but shot no solid bolt. Mere violent rant, \& then broke off. We listened in to the end. A savage howl like a person excruciated; then howls from the audience; then a more spaced \& measured sentence. Then another bark. Cheering ruled by a stick. Frightening to think of the faces. \& the voice was frightening. But as it went on we said (only picking a word or two) anti-climax. This seems to be the general verdict. He darent cross the line. Comes up to it \& stands bawling insults », Virginia Woolf, Diary, vol. V, op.cit, p. 169.

17. Virginia Woolf, Journal intégral, op.cit., p. 1346, extrait traduit de «I went to the London library. Sat in the basement \& looked up the Times on PIP [Post Impressionism] in 1910. Old sweeper gently dusting. Came \& said, They're telling us to try our masks. Have you got yours I asked. No not yet. And shall we have war? I fear so, but I still hope not. I live out in Putney. Oh they've laid in sand bags; the books will be moved; but if a bomb strikes the house... May I dust under your chair? », Virginia Woolf, Diary, vol. V, op. cit., p. 174. 
gaz. Les « effets d'intime ${ }^{18}$ » visent à susciter l'émotion : chuchotement, tête-à-tête, simplicité et spontanéité des propos créent une atmosphère d'authenticité comme rempart contre le mensonge de la parole publique. Or cette scène qui associe l'ardeur au travail du balayeur à l'expression de la bonté humaine a justement lieu dans une bibliothèque, figure architecturale et culturelle d'une politique qui a longtemps exclu les femmes, symbole même de la tradition culturelle et de la pérennité de l'histoire. Paradoxalement, la question de la grandeur et de la mémoire de la littérature n'est plus le problème des grands hommes, mais des petites gens, et le mouvement de l'histoire ne relève plus du collectif, de la communauté politique mais des individus avec leur sensibilité personnelle. On pense bien sûr aux toiles de Sickert où les femmes nues dans des chambres portent en elles toute la fragilité et l'usure de l'histoire : ces tableaux remplacent les gigantesques portraits ou autres fresques auxquels nous avait habitués Delacroix ou Géricault. Comme Sickert, Virginia retire les oripeaux épiques pour ne laisser que les petits faits banals : elle nous donne à lire une histoire humanisée, vécue de l'intérieur. Et si l'appréhension de l'histoire s'en trouve modifiée, c'est aussi le petit fait insignifiant qui en est poétisé, mythifié : dans le bref échange de la British Library, Woolf renouvelle les pratiques politiques et donne à lire une allégorie des valeurs de l'humanité cristallisées autour de la bonté, de l'individu et du dialogue. C'est cette foule de petites gens que met en scène Woolf et dont la parole réconfortante, inquiète et humble vient contredire l'horreur des vociférations hitlériennes et la fausseté des discours des hommes politiques.

L'écriture du journal est donc un lieu de lutte contre le discours dominant et tyrannique, mais aussi le lieu de construction d'un discours autre. En refusant les diverses pratiques de théorisation, de modélisation et d'abstraction, incapables d'expliciter la complexité de la réalité politique, Woolf met en place une pensée hors système, aléatoire, qui accueille les contradictions et les fluctuations d'un moi instable et d'une expérience historique vive. Elle récuse alors toute une tradition occidentale de la prose politique, qu'il s'agisse de la rhétorique ordonnée et argumentative, héritée de Cicéron, ou de l'essai philosophique. La forme fragmentaire, discontinue et inachevable du journal renouvelle le dire politique et la conception de la société qui va avec. L'écriture du quotidien qui décompose la réflexion juxtapose et disperse des vérités éclatées parfois contradictoires. D'ailleurs, dans sa matérialité même, le texte signifie l'incomplétude : le mode fragmentaire du journal troué de multiples blancs et ellipses est symptomatique des failles de la pensée. Woolf substitue alors à une conception surplombante, globale et distanciée, une vision parcellaire et partielle qui vise à dire le non-sens de la politique comme science humaine et comme

18. Brigitte et José-Luis Diaz parlent de « l'effet d'intime » dans « Le siècle de l'intime », Itinéraires. Littérature, textes, cultures, $\mathrm{n}^{\circ}$ 2009-4, « Pour une histoire de l'intime et de ses variations », p. 122. 
pratique signifiante. Par ailleurs, les tâtonnements et les réitérations participent d'une esthétique de la répétition : " No war yet anyhow ${ }^{19}$ », « Not at war yet ${ }^{20} »$, « This may be the last day of peace ${ }^{21} »$, « This is I suppose the last hour of peace ${ }^{22} »$, autant de piétinements qui disent dans une ultime plainte litanique la crise de la conception morale et platonicienne de la gouvernance politique. Pensée en mouvement, refus de fixation du sens dans la clôture et discours oblique se substituent à la ligne droite et logique de la tradition des penseurs et favorisent l'idée d'une société en perpétuelle évolution. Woolf écrit «par lampées et par saccades » dans une forme très montaigniste qui n'a « pas de soudure, pas de mise au point, résultat peutêtre d'une affectation de non-conformisme ${ }^{23} \gg$. Cette poétique de la rature, du « griffonnage » ( « scribble $\left.{ }^{24} »\right)$ privilégie le sensible et laisse une large place à la fantaisie. En construisant une parole vive, affective et nouvelle, Woolf donne à son journal une visée pragmatique et vitale :

This book will serve to accumulate notes, the fruit of such quickenings. And for the 100th time I repeat - any ideas more real than any amount of war misery. And what one's made for. And the only contribution one can make. This little pitter patter of ideas is my whiff of shot in the cause of freedom - so I tell myself, thus bolstering up a figment - a phantom: recovering that sense of something pressing from outside which consolidates the mist, the non-existent ${ }^{25}$,

écrit-elle pour souligner le lien intrinsèque entre écriture et révolte.

Il serait donc simplificateur de lire le Journal comme la seule antichambre des pamphlets. Au contraire, l'écriture personnelle contribue

19. Virginia Woolf, Diary, vol. V, op. cit., p. 169, traduit par « toujours est-il que ce n'est pas encore la guerre », Journal Intégral, op. cit., p. 1341.

20. Ibid., p. 232, traduit par « Nous ne sommes toujours pas en guerre », Journal intégral, op. cit., p. 1400.

21. Ibid., p. 174, traduit par « C'est peut-être aujourd'hui le dernier jour de paix », Journal intégral, op. cit., p. 1345.

22. Ibid., p. 233, traduit par « Nous vivons très probablement, je présume, notre dernière heure de paix », Journal intégral, op. cit., p. 1401.

23. Virginia Woolf, Journal intégral, op. cit., p. 942, traduction de «[...] I write this diary in gulps $\&$ jerks; $\&$ has not the strength to come down in one blow: no welding, no shaping - the result of a false anti-literariness perhaps ", Diary, vol. IV, op. cit., p. 95.

24. Le verbe « scribble» et ses polyptotes servent à définir la forme décousue et spontanée de l'écriture du journal qui nous fait passer sans transition d'un sujet à l'autre: « this unpremetidated scribbling », Virginia Woolf, Diary, vol. I, op. cit., p. 304 (« ce griffonnage improvisé », Journal intégral, op.cit., p. 284).

25. Virginia Woolf, Diary, vol. V, op. cit., p. 235, traduit par « Ce cahier va me servir à accumuler des notes - les fruits de ce genre de remise en route. Et pour la énième fois je le redis : toute idée, quelle qu'elle soit, contient plus de réalité que tous les maux engendrés par la guerre. Ce pour quoi nous avons été créés; et notre seule contribution possible... Ce petit crépitement d'idées est une salve que je tire au nom de la liberté - voilà, du moins, ce que je me dis », Journal intégral, op. cit., p. 1404. 
à la genèse d'une voix indépendante et revendicative qui se déploie dans et par l'intime de l'écriture. Ce n'est d'ailleurs pas anodin si Woolf s'empare du genre féminin par excellence pour créer son discours politique et transgresser les interdits qui pèsent sur la femme. C'est une écriture du dedans, marginale, qui est valorisée contre la logique rationnelle des discours masculins institués. Le Journal actualise la naissance d'une « écriturefemme ${ }^{26} \gg$ de l'histoire, d'une manière féminine de penser et de dire la politique. Virginia Woolf prend la parole, s'octroie le droit de parler d'elle et de se poser en fondement d'une vision qui lui est propre. Nouvelle forme de discours engagé, le Journal devient une véritable " forme-sens » où l'émergence du moi, la présence de scènes intimistes, la valorisation de l'affect sont autant de manières de lutter contre le système institué, son fonctionnement et ses dirigeants. Cette refonte formelle permet de redéfinir les concepts a priori antinomiques de politique et d'intime. Ce dernier permet de repenser le politique et ses attributs. Woolf quitte la sphère de la survalorisation de valeurs politiques ancestrales - la tradition, le culte de la raison et de la guerre, le patriotisme -, pour redonner une place au sensible, à l'individu avec ses zones d'ombre et ses failles, et en particulier à la femme. Réactualisant le mythe d'Antigone ${ }^{27}$, elle invente ce qu'elle appelle "the Outsiders Society », la "société des Marginales », dans Trois Guinées : composée de filles d'hommes cultivés, qui revendiquent leur indépendance financière et leur liberté d'expression, cette société se propose de lutter contre la guerre et les fascismes non par les armes, mais par le développement de la potentialité affective. Dès lors, le Journal peut se lire comme l'actualisation de ce discours féminin et contestataire, comme un lieu de révolte où l'originalité, la liberté de dire et l'intimité sont revendiquées comme des droits et comme des solutions alternatives à la société et la politique actuelles. Au principe d'action se substitue celui de l'écoute et de l'émotion, à la Chambre des députés, lieu paroxystique de la représentation et du spectacle, s'oppose la « chambre à soi ${ }^{28}$ ", espace de déploiement d'une parole libérée et d'une pensée autonome. Avec son Journal, Woolf donne voix aux marginaux, et devient un auctor au sens latin du terme, c'est-à-dire une voix à l'origine d'un discours d'autorité : le discours non conventionnel de la femme, du journal, se transforme en discours d'autorité au sein même de la marginalité. Elle met alors en place

26. Béatrice Didier, L'Écriture-femme, Paris, PUF, 1991. Selon Béatrice Didier, il existe des lignes de force communes qui permettent de reconnaître un écrit féminin. Ainsi l'écriture féminine serait liée au genre du « moi » : «l'écriture féminine est une écriture du Dedans », ibid., p. 37.

27. Le mythe d'Antigone est récurrent dans les écrits woolfiens. Son opposition à Créon, son refus des lois humaines en faveur de la loi divine, en font le symbole de la lutte contre toute tyrannie. Cette figure exemplaire permet à Woolf de confronter un système éthique féminin basé sur le sentiment à la rationalité masculine en lutte.

28. Une chambre à soi est le titre d'un des pamphlets de Woolf, traduction de A Room of One's Own. 
un espace de l'entre-deux, entre l'intime et le politique, entre le « je » et le «nous ", la sensibilité et l'intelligence critique, le mythe personnel et l'archétype, la marginalité et la communauté, espace qui refuse de se plier à la cartographie sanctifiée par l'histoire patriarcale.

Sandra Cheilan

Université Paris Ouest Nanterre La Défense Centre de recherches Littéraure et poétique comparées (EA 3931) 